\title{
POSTER
}

\section{Lichen plan buccal induit par Mitotane : à propos d'un cas}

\section{Herry $\mathbf{H}^{1}$, SchmoucŠovitch $A^{1}$, Thuillier $\mathrm{P}^{2}$, Kerlan $\mathbf{V}^{3}$, Le Toux $G^{4}$, Boisramé $\mathrm{S}^{4}$}

1 - Interne en médecine bucco-dentaire, Service d'odontologie, CHRU de Brest

2 - CCA, Service d'endocrinologie, CHRU de Brest

3 - PU-PH, Service d'endocrinologie, CHRU de Brest

4 - MCU-PH, Service d'odontologie, département de chirurgie orale, CHRU de Brest

Le lichen plan buccal est une dermatose cutanéo-muqueuse fréquente qui touche 1 à $2 \%$ de la population adulte et principalement les femmes (ratio 4 femmes pour 1 homme). La symptomatologie clinique est variée : érythémateuse, érosive ou bulleuse. Le lichen peut être quiescent ou évolutif. L'étiopathogénie est diverse : forme psychosomatique ou secondaire (associée à une pathologie générale, secondaire à la maladie du greffon contre l'hôte). On retrouve également des lichens plans induits (formes médicamenteuses ou toxiques). (1)

Le cortico-surrénalome malin est un cancer de la glande surrénale qui se développe aux dépens de la couche externe de cette glande. C'est une tumeur rare (1 à 2 cas sur 1 million par an). Elle survient le plus souvent chez l'adulte entre 40 et 50 ans mais on observe également un pic d'incidence chez l'enfant de moins de 15 ans. Cette tumeur est observée le plus souvent chez la femme. (2)

Le cas rapporté est celui d'une femme âgée de 50 ans suivie dans le service d'endocrinologie pour une maladie de Cushing qui s'est compliquée en un cortico-surrénalome malin traité en décembre 2013 par surrénalectomie. Une chimiothérapie anti-surrénalienne par mitotane (Lysodren ${ }^{\circledR}$ ) a été instaurée en janvier 2014. En septembre 2014 la patiente rapporte une gêne avec des douleurs au niveau des muqueuses vaginales et buccales. Un premier diagnostic de candidose buccale a été évoqué mais sans amélioration au traitement par fluconazole (Triflucan ${ }^{\circledR}$ ). La patiente a alors été adressée à la consultation pluridisciplinaire du lupus du service de rhumatologie mais les examens cliniques et immunologiques n'étaient pas en faveur d'un lupus induit. En parallèle une biopsie a été réalisée, ces lésions se sont révélées être un lichen plan érosif. Ce lichen est depuis plus d'un an résistant aux corticoïdes ainsi qu'à la ciclosporine et l'acitrétine. (3)

Devant cet état réfractaire à tout traitement elle a été adressée en consultation de chirurgie et pathologie orale du service d'odontologie en novembre 2015. À l'examen clinique la patiente présentait un lichen érosif jugal droit et gauche compliqué d'un botryomycome cutané rétro commissural gauche, ainsi qu'un lichen plan érosif sur le bord latéral droit de la langue. La patiente signale également des lésions similaires au niveau vulvaire.

Lors de l'entretien, la patiente rapporte que l'apparition des lésions date de six mois après l'instauration du traitement par mitotane.

Une déclaration de pharmacovigilance a alors été réalisée. Une discussion est actuellement en cours et le dossier sera vu en consultation pluridisciplinaire afin d'évaluer le rapport bénéficerisque quand au maintien ou non du traitement par mitotane, ce dernier ne pouvant être actuellement substituable. 